\title{
Multimode Phenomena in Hard Oscillators Coupled by a Time-Varying Resistor
}

\author{
Masaaki Kojima $^{\dagger *}$, Yoko Uwate ${ }^{\dagger}$ and Yoshifumi Nishio ${ }^{\dagger}$ \\ $†$ Department of Electrical and Electronic Engineering, Tokushima University \\ 2-1 Minami-Josanjima, Tokushima, 770-8506 Japan \\ † Engineering Administration Department, NHK \\ 2-2-1 Jinnan, Shibuya-ku, Tokyo, 150-8001 Japan \\ Email: \{kojima, uwate, nishio\}@ee.tokushima-u.ac.jp
}

\begin{abstract}
We investigate multiple circuits with fifthpower nonlinear characteristics coupled by a time-varying resistor. In particular, we confirm some different types of double-mode (simultaneous asynchronous) oscillations which do not occur for the systems with third-power nonlinear characteristics.
\end{abstract}

\section{Introduction}

Coupled oscillator systems can be good models to describe various nonlinear phenomena in the field of natural sciences, neural systems and communication networks. The negative resistor within the oscillator has fifth-power $v-i$ characteristics. As a result, the oscillator is known to exhibit hard excitation. The oscillator converges on the origin for small initial values because fifth-power characteristics near the origin act as a positive resistor. Greater initial values than the origin stable regions are required to generate the oscillation. Such an oscillator is called a hard oscillator or that with hard nonlinearity. Datardina and Linkens have investigated two van der Pol oscillators with hard nonlinearities coupled by an inductor [1]. They have confirmed that four different modes, which are zero, in-phase synchronous mode, antiphase synchronous mode and double-mode, coexist for some range of parameter values. These modes are zero (non-oscillation), two single-modes (in-phase and antiphase synchronizations), and double-mode (simultaneous asynchronous oscillations with in-phase and anti-phase frequency components). In particular, the double-mode oscillations are stably excited although they do not occur for the case of the common negative resistor with thirdpower characteristics. Furthermore, Endo and Ohta have confirmed that some different types of single-modes and double-modes in two, three and four van der Pol oscillators with hard nonlinearities coupled by inductors are stably excited [2].

On the basis of these studies, we have proposed two coupled chaotic circuits with hard nonlinearities for the purpose of looking for interesting phenomena as doublemode chaotic oscillations [3][4]. We have confirmed double-mode chaos with one-periodic in-phase and chaotic anti-phase single-modes [3] and that with torus and chaos [4], respectively. We have also confirmed the other type of double-mode chaos with one-periodic inphase and chaotic three-phase single-modes in three coupled chaotic circuits with hard nonlinearities [5]. However, we have not found that with chaos and chaos in these systems. We are considering the reason that these phenomena do not exist could be caused by frequency differences between two single-modes.

On the other hand, we have investigated synchronization phenomena of two van der Pol oscillators coupled by a time-varying resistor which means positive and negative resistors switching periodically [6]. We have confirmed that in-phase and anti-phase single-modes are stably excited in these systems at the same parameter values. We have also confirmed that two chaotic singlemodes coexist in an improved chaos version of these systems [7]. The frequency differences between two different single-modes in the systems coupled by a timevarying resistor are possibly smaller than those in the systems coupled by an inductor or capacitor such as the previous systems. Therefore, we can expect to improve the fields of the double-mode chaos [3]-[5] if we find doublemode oscillations in hard oscillators coupled by a timevarying resistor. The double-mode oscillation in the systems coupled by a time-varying resistor has not been reported yet while the coexistence of the each single-mode oscillation is confirmed [6][7]. In this study, we propose multiple van der Pol oscillators with hard nonlinearities coupled by a time-varying resistor and confirm some different types of double-mode oscillations in these systems.

\section{Circuit Model}

Figure 1 shows the proposed circuit model. In this model, $N$ identical van der Pol oscillators with hard nonlinearities are coupled by a time-varying resistor (TVR). The time waveform characteristics of the TVR are shown in Fig. 2. The parameters $\omega_{t}$ and $p$ mean the angular frequency and the duty ratio, respectively. The TVR is realized by a square wave of switching positive and negative resistors.

The equations governing the circuit in Fig. 1 are described as follows: 


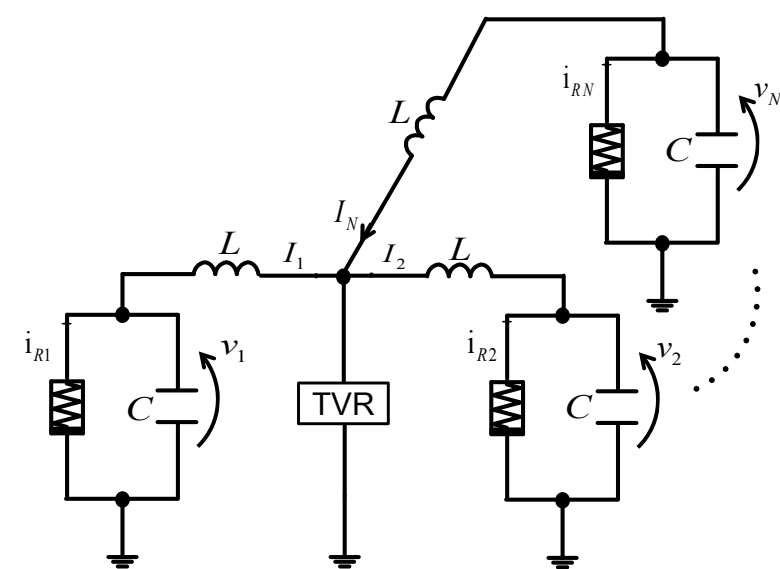

Figure 1: Circuit model (TVR stands for time-varying resistor).

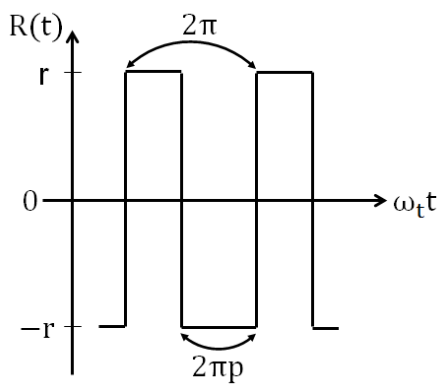

Figure 2: TVR characteristics.

$$
\begin{gathered}
C \frac{d v_{k}}{d t}=-i_{k}-i_{R k}\left(v_{k}\right) \\
L \frac{d i_{k}}{d t}=v_{k}-\mathrm{R}(\mathrm{t}) \sum_{j=1}^{N} i_{j} \\
(k=1,2, \cdots, N)
\end{gathered}
$$

$i_{R k}\left(v_{k}\right)$ indicates $v-i$ characteristics of the fifth-power nonlinear resistor. It is approximated by the following function:

$$
i_{R k}\left(v_{k}\right)=g_{1} v_{k}-g_{3} v_{k}^{3}+g_{5} v_{k}^{5}\left(g_{1}, g_{3}, g_{5}>0\right) .
$$

By changing the variables and the parameters,

$$
\begin{aligned}
& v_{k}=\sqrt[4]{\frac{g_{1}}{5 g_{5}}} x_{k}, \quad i_{k}=\sqrt{\frac{C}{L}} \sqrt[4]{\frac{g_{1}}{5 g_{5}}} y_{k}, \\
& \beta=\frac{3 g_{3}}{g_{1}} \sqrt{\frac{g_{1}}{5 g_{5}}}, \gamma=\mathrm{r} \sqrt{\frac{C}{L}}, \quad \varepsilon=g_{1} \sqrt{\frac{L}{C}} \\
& \mathrm{t}=\sqrt{L C} \tau, \quad \omega=\frac{1}{\sqrt{L C}} \omega_{\mathrm{t}}, " . "=\frac{d}{d \tau},
\end{aligned}
$$

(1) is normalized as

$$
\dot{x}_{k}=-y_{k}-\varepsilon\left(x_{k}-\frac{1}{3} \beta x_{k}^{3}+\frac{1}{5} x_{k}^{5}\right)
$$

$$
\begin{gathered}
\dot{y}_{k}=x_{k}-\Gamma(\tau) \sum_{j=1}^{N} y_{j} \\
(k=1,2, \cdots, N)
\end{gathered}
$$

$\Gamma(\tau)$ corresponds to the normalized characteristics of the TVR as shown in Fig. 3.

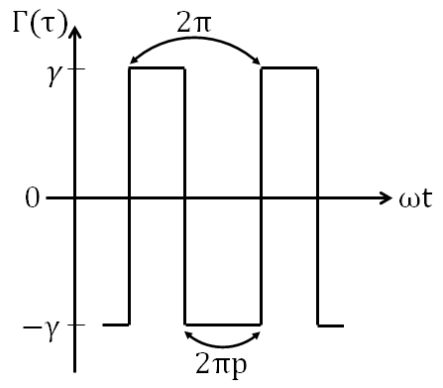

Figure 3: Normalized TVR characteristics.

\section{Multi-Mode Oscillations}

\subsection{Two Coupled Oscillators}

In this section, we mention two coupled oscillators with hard nonlinearities in the case of $N=2$ in Fig. 1 . In this system, four different oscillation modes (zero, in-phase single-mode, anti-phase single-mode and double-mode) coexist. These modes are determined by the initial values. Figures 4 and 5 show some simulation results on each mode attractor and time waveform, respectively. The parameter values in Figs. 4 and 5 are $\beta=3.2, \gamma=$ $0.1, \varepsilon=0.01, \omega=1.5$ and $p=0.5$.

Zero means neither of the two oscillators is excited. This state is always stable in some range of small initial values. We do not mention this state in the rest of this manuscript because it is trivial.

In-phase single-mode means that the two oscillators are synchronized with the in-phase. As the simulation results in Figs. 4-(1) and 5-(1) show, they are stably excited with a 0 phase difference.

Anti-phase single-mode means that the two oscillators are synchronized with the opposite phase. As the simulation results in Figs. 4-(2) and 5-(2) show, they are stably excited with a $\pi$ phase difference.

Double-mode means that the above two single-mode oscillations are excited simultaneously and asynchronously. As the simulation results in Figs. 4-(3) and 5-(3) show, they are simultaneous asynchronous oscillations with in-phase and anti-phase single-modes at the stable. The envelopes of the double-mode time waveform are synchronized with the opposite phase in Fig. 5-(3).

\subsection{Three Oscillators}

Here is the case of $N=3$ in Fig. 1. It is also four different oscillation modes (zero, in-phase single-mode, 


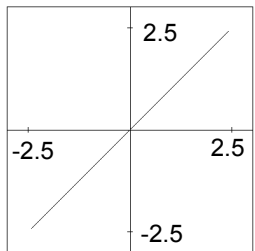

(a) $\left(x_{1}-x_{2}\right)$

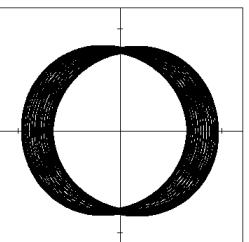

(b) $\left(x_{1}-y_{1}\right)$

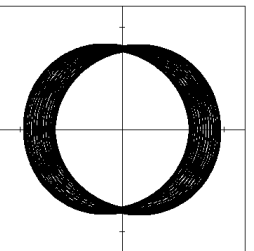

(c) $\left(x_{2}-y_{2}\right)$

(1) In-phase single-mode

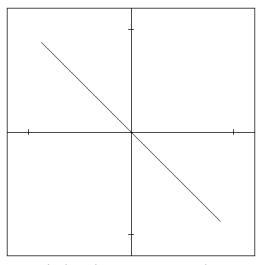

(a) $\left(x_{1}-x_{2}\right)$

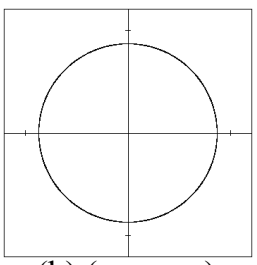

(b) $\left(x_{1}-y_{1}\right)$

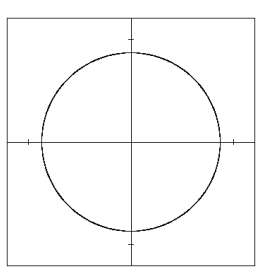

(c) $\left(x_{2}-y_{2}\right)$

(2) Anti-phase single-mode

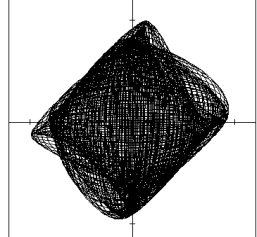

(a) $\left(x_{1}-x_{2}\right)$

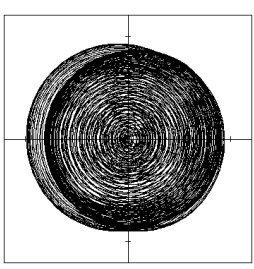

(b) $\left(x_{1}-y_{1}\right)$

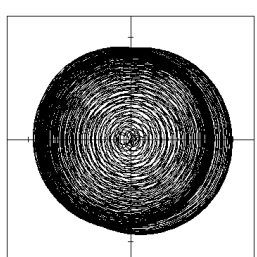

(c) $\left(x_{2}-y_{2}\right)$

(3) Double-mode

Figure 4: Attractors of three different oscillation modes $(N=2)$.

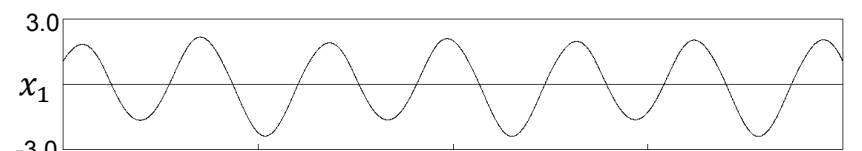

$-3.0$

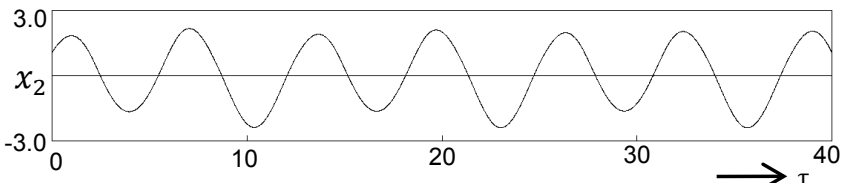

(1) In-phase single-mode
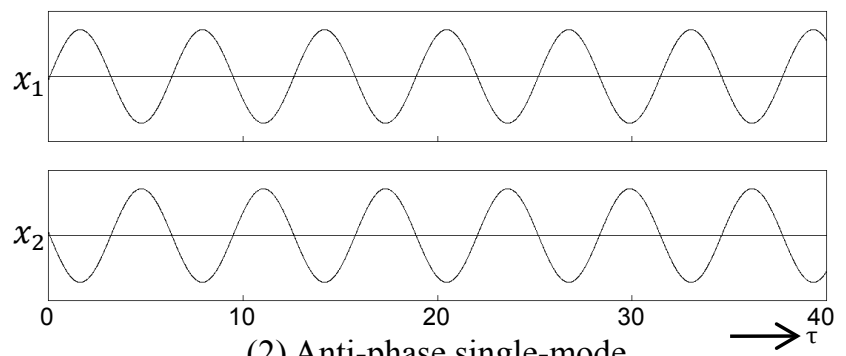

(2) Anti-phase single-mode
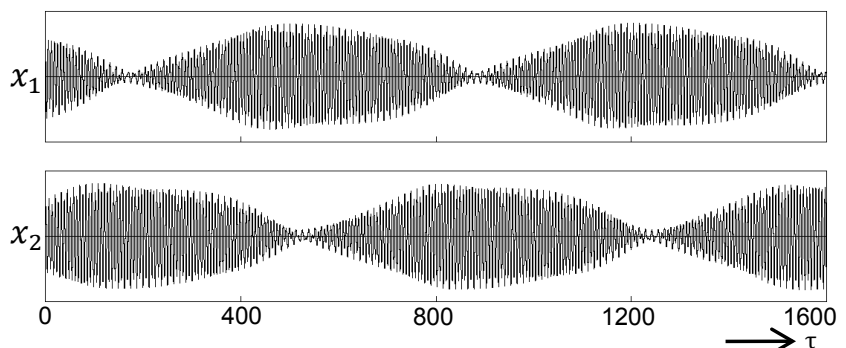

(3) Double-mode

Figure 5: Time waveforms vs amplitude $(N=2)$.

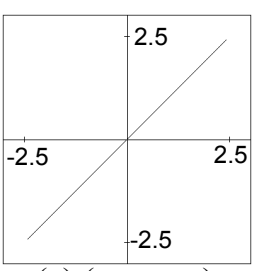

(a) $\left(x_{1}-x_{2}\right)$

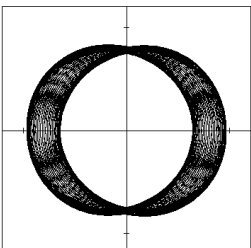

(d) $\left(x_{1}-y_{1}\right)$

(1) In-phase single-mode

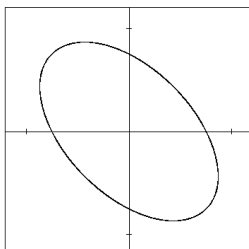

(a) $\left(x_{1}-x_{2}\right)$

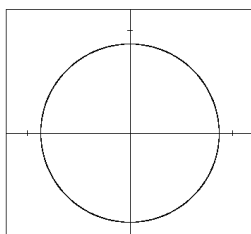

(d) $\left(x_{1}-y_{1}\right)$

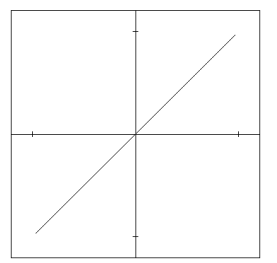

(b) $\left(x_{1}-x_{3}\right)$

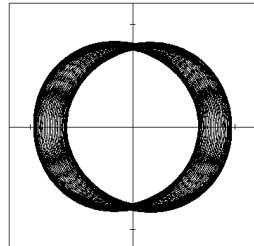

(e) $\left(x_{2}-y_{2}\right)$

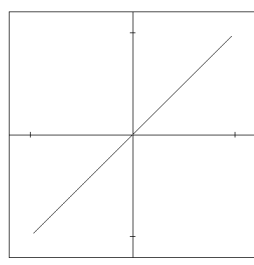

(c) $\left(x_{2}-x_{3}\right)$

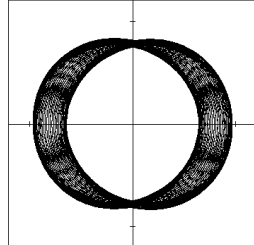

(f) $\left(x_{3}-y_{3}\right)$

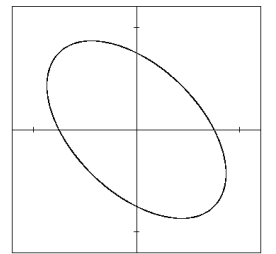

(b) $\left(x_{1}-x_{3}\right)$

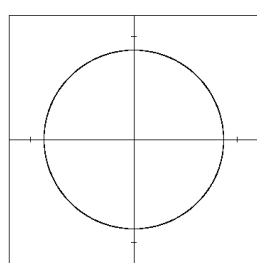

(e) $\left(x_{2}-y_{2}\right)$

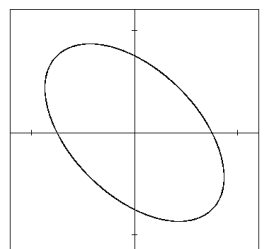

(c) $\left(x_{2}-x_{3}\right)$

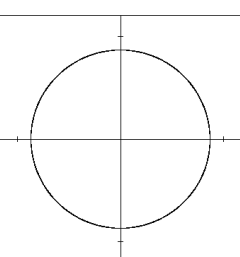

(f) $\left(x_{3}-y_{3}\right)$
(2) Three-phase single-mode

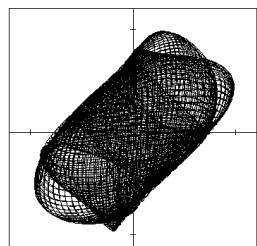

(a) $\left(x_{1}-x_{2}\right)$

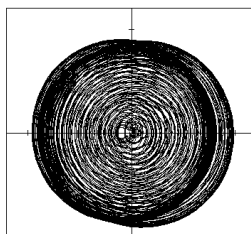

(d) $\left(x_{1}-y_{1}\right)$

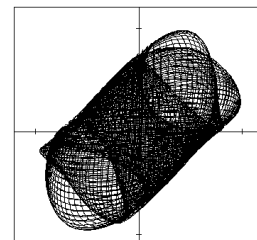

(b) $\left(x_{1}-x_{3}\right)$

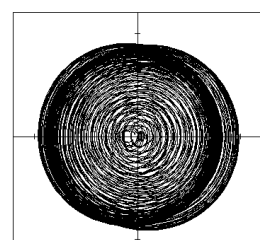

(e) $\left(x_{2}-y_{2}\right)$

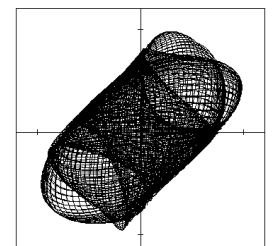

(c) $\left(x_{2}-x_{3}\right)$

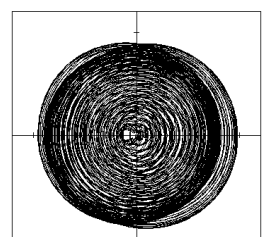

(f) $\left(x_{3}-y_{3}\right)$
(3) Double-mode

Figure 6: Attractors of three different oscillation modes $(N=3)$.

three-phase single-mode and double-mode) coexist. Figures 6 and 7 show some simulation results on each mode attractor and time waveform, respectively. The parameter values in Figs. 6 and 7 are $\beta=3.2, \gamma=$ $0.07, \varepsilon=0.01, \omega=1.5$ and $p=0.5$. 


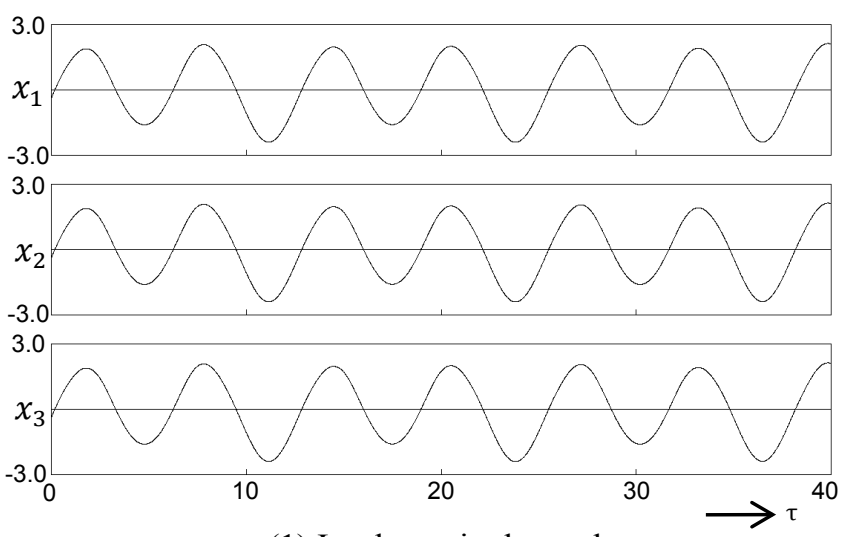

(1) In-phase single-mode
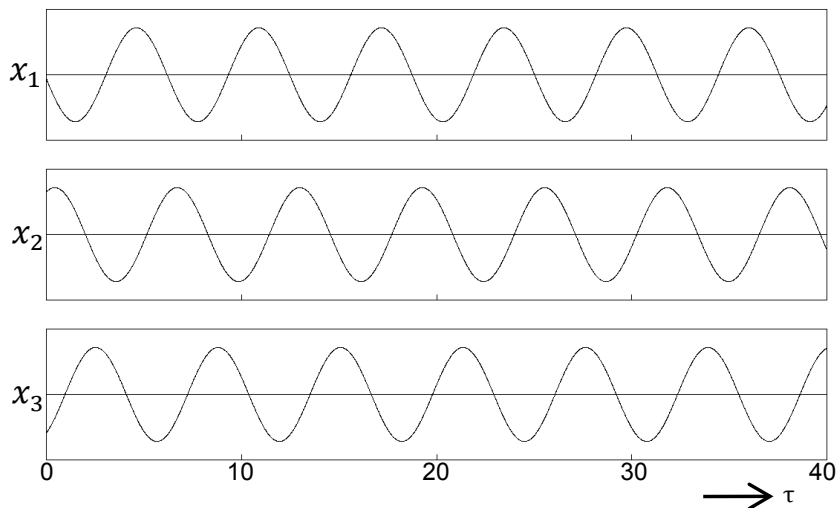

(2) Thee-phase single-mode
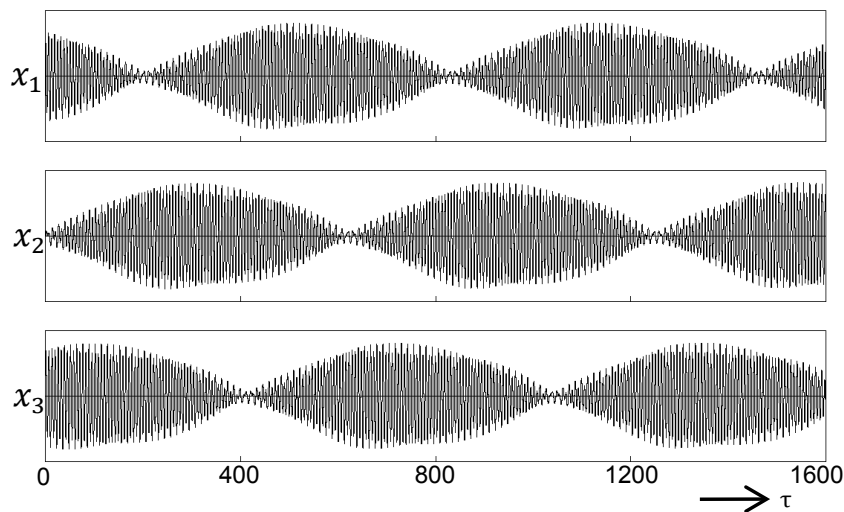

(3) Double-mode

Figure 7: Time waveforms vs amplitude $(N=3)$.

Zero means that three oscillators are not excited.

In-phase single-mode means that the three oscillators are synchronized with the in-phase. As the simulation results in Figs. 6-(1) and 7-(1) show, they are stably excited with a 0 phase difference.

Three-phase single-mode means that the three oscillators are synchronized with a $2 \pi / 3$ phase difference. As the simulation results in Figs. 6-(2) and 7-(2) show, they are stably excited with a $2 \pi / 3$ phase difference.

Double-mode means that the above two single-mode oscillations are excited simultaneously and asynchronously. As the simulation results in Figs. 6-(3) and 7-(3) show, they are simultaneous asynchronous oscillations with in-phase and three-phase single-modes at the stable. The envelopes of the double-mode time waveform are synchronized with a $2 \pi / 3$ phase difference in Fig. 7-(3).

\section{Conclusions}

We proposed two and three oscillators with hard nonlinearities coupled by a time-varying resistor and looked for the parameter values that two different singlemodes and a double-mode could coexist and be stably excited. As a result, we confirmed that these modes coexisted and were stably excited by computer simulations. Especially, the double-mode oscillations for the systems coupled by a time-varying resistor were the first report. We will also investigate chaotic circuits with hard nonlinearities coupled by a time-varying resistor in the near future.

\section{References}

[1] S. P. Datardina and D. A. Linkens, "Multimode oscillations in mutually coupled van der Pol type oscillators with fifth-power nonlinear characteristics," IEEE Trans. Circuits Syst., vol.25, no.5, pp.308-315, May 1978.

[2] T. Endo and T. Ohta, "Multimode oscillations in a coupled oscillator system with fifth-power nonlinear characteristics," IEEE Trans. Circuits Syst., vol.27, no.4, pp.227-283, Apr. 1980.

[3] Y. Nishio and A. Ushida, "Multimode chaos in two coupled chaotic oscillators with hard nonlinearities," IEICE Trans. Fundamentals, vol.79-A, no.2, pp.227-232, Feb. 1996.

[4] M. Kojima, M. Yamauchi, Y. Nishio and A. Ushida, "Simultaneous asynchronous oscillation of chaos and torus in coupled chaotic circuits," Proc. NOLTA '00, vol.2, pp.567-570, Sep. 2000.

[5] M. Kojima, Y. Nishio and A. Ushida, "Simultaneous asynchronous chaotic oscillation of in-phase and threephase in coupled chaotic circuits," Proc. ITC-CSCC '01, vol.2, pp.716-719, July 2001.

[6] Y. Uwate and Y. Nishio, "Synchronization phenomena in van der Pol oscillators coupled by a time-varying resistor," International Journal of Bifurcation and Chaos, vol.17, no.10, pp.3565-3569, Oct. 2007.

[7] Y. Uwate and Y. Nishio, "Switching phase states of chaotic circuits coupled by a time-varying resistor," Proc. of ISCAS '07, pp.209-212, May 2007. 